\title{
Improved $\gamma$-linolenic acid production in Mucor circinelloides by homologous overexpressing of delta-12 and delta- 6 desaturases
}

Yao Zhang ${ }^{1 \dagger}$, Xiao Luan ${ }^{2 \dagger}$, Huaiyuan Zhang ${ }^{1}$, Victoriano Garre ${ }^{3}$, Yuanda Song ${ }^{1,2^{*}}$ and Colin Ratledge ${ }^{4}$

\begin{abstract}
Background: $\gamma$-Linolenic acid (GLA) is important because of its nutritional value and medicinal applications. Although the biosynthetic pathways of some plant and microbial GLA have been deciphered, current understanding of the correlation between desaturases and GLA synthesis in oleaginous fungi is incomplete. In previous work, we found that a large amount of oleic acid (OA) had not been converted to linoleic acid (LA) or GLA in Mucor circinelloides CBS 277.49, which may be due to inadequate activities of the delta-12 or delta- 6 desaturases, and thus leading to the accumulation of OA and LA. Thus, it is necessary to explore the main contributing factor during the process of GLA biosynthesis in M. circinelloides.
\end{abstract}

Results: To enhance GLA production in M. circinelloides, homologous overexpression of delta-12 and two delta- 6 desaturases (named delta-6-1 and delta-6-2, respectively) were analyzed. When delta- 6 desaturase were overexpressed in M. circinelloides, up to 43\% GLA was produced in the total fatty acids, and the yield of GLA reached $180 \mathrm{mg} / \mathrm{l}$, which were, respectively, 38 and $33 \%$ higher than the control strain.

Conclusion: These findings revealed that delta-6 desaturase (especially for delta-6-1 desaturase) plays an important role in GLA synthesis by M. circinelloides. The strain overexpressing delta-6-1 desaturase may have potential application in microbial GLA production.

Keywords: Delta-6 desaturase, Delta-12 desaturase, GLA production, Homologous overexpression, Mucor circinelloides

\section{Background}

$\gamma$-Linolenic acid (GLA, 18:3, delta-6,9,12) is an important $\mathrm{n}-6$ polyunsaturated fatty acid (PUFA) that has many health and medicinal effects for prevention and treatment of inflammatory disorders, diabetes, cardiovascular disorders, cancers, and some other diseases [1-4]. The traditional sources of GLA are from a relatively small number

\footnotetext{
*Correspondence: ysong@sdut.edu.cn

${ }^{\dagger}$ Yao Zhang and Xiao Luan contributed equally to this work ${ }^{1}$ Colin Ratledge Center for Microbial Lipids, School of Agricultural Engineering and Food Science, Key Laboratory of Shandong Provincial Universities for Technologies in Functional Agricultural Products, Shandong University of Technology, 266 Xincun West Road, Zibo, Shandong 255000, People's Republic of China

Full list of author information is available at the end of the article
}

of plant seeds, including evening primrose (Oenothera biennis L.), borage (Borago officinalis L.) and blackcurrant (Ribes nigrum L.) [5-7] where the content of GLA may be up to $22 \%$ of the total fatty acids. However, GLA production from plant seeds is easily influenced by region, climate and seasons, resulting in variable quantities and qualities of the oils. Genetically-engineered safflower seeds are now available in which GLA may now be up to $70 \%$ of the total fatty acids $[8,9]$ but, because of the uncertainties surrounding the acceptability of genetically modified (GM) materials as neutraceuticals, there is continued interest in developing microbial oils over conventional sources. Thus, a promising alternative source comes from oleaginous microorganisms with high GLA contents [5, 10-12]. 
Generally in plant and microbial cells, the biosynthesis of PUFA derives from saturated stearic acid (18:0), which is first converted by delta- 9 desaturase to yield oleic acid (OA, 18:1, delta-9). Then, delta-12 desaturase converts OA to linoleic acid (LA, 18:2 delta-9, 12). Subsequently, GLA is synthesized by introducing a double bond into LA using a delta- 6 desaturase. Thus, three desaturases, the delta-9, delta-12 and delta-6, accomplish the desaturation processes in GLA synthesis [5, 13, 14]. Genes coding for those desaturases have been cloned from diverse organisms ranging from prokaryotes to higher eukaryotes, and overexpressed in several hosts, including microalgae, yeasts and plants $[8,13-16]$. However, few researches on homologous expression of fungal desaturases have been reported.

Mucor circinelloides, a typical oleaginous filamentous fungus, has been widely used to investigate GLA production since the 1980s $[12,17]$. Previously, we found that the proportion of OA, LA and GLA in total fatty acids of $M$. circinelloides were 38,11 , and $19 \%$, respectively $[18,19]$. This suggested that a large amount of OA had not been converted to LA or GLA, which may be due to inadequate activities of the delta- 12 or delta- 6 desaturases, and thus leading to the accumulation of $\mathrm{OA}$ and LA. Thus, it is necessary to explore the main contributing factor during the process of GLA biosynthesis in $M$. circinelloides. In this study, we report the cloning and homologous overexpression of the delta- 12 and delta- 6 desaturases derived from $M$. circinelloides itself with the aim of determining the correlation between fatty acid desaturases and GLA biosynthesis in oleaginous fungi.

\section{Results and discussion}

\section{Construction of delta-12 and delta- 6 desaturase genes overexpressing strains}

Based on the genomic data of $M$. circinelloides CBS 277.49 , we found one delta-12 desaturase gene, named D12 (JGI accession number ID155268, $1351 \mathrm{bp}$ ), and two delta-6 (delta-6-1 and delta-6-2) desaturase genes, named D61 (JGI accession number ID37214, 1738 bp) and D62 (JGI accession number ID105367, 1575 bp). To determine if the delta- 12 and delta- 6 desaturases were involved in fatty acid accumulation, overexpressing strains of these genes were generated. The genes, $D 12$, D61 and D62, were cloned from the genome of M. circinelloides and subsequently inserted into the expression vector, pMAT1552, that contains the strong zrt1 promoter, the pyrG gene as a selectable marker and flanking sequences corresponding to regions surrounding the carotenogenic carRP gene to allow its chromosomal integration by homologous recombination (Fig. 1a). The D12, D61 and D62 overexpressing plasmids, pMAT1552-D12, pMAT1552-D61 and pMAT1552-D62, were transformed into the recipient strain MU402 and transformation and selection of the colonies were carried out as described by [20]. Three overexpressing and control transformants were selected at random and then confirmed by PCR analysis. Amplification was carried out using a primer pair (P3-F/R) that amplified the desaturase gene and $1000 \mathrm{bp}$ sequences of the plasmid pMAT1552. Thus, the corresponding D12 (2736 bp), D61 (2351 bp) and D62 (2575 bp) gene fragments were amplified and, as expected, resulted in transformants genomes, whereas no desaturase gene fragment was amplified in the control strain (Mc-1552 carring the vector pMAT1552) and wild-type strain (M. circinelloides CBS 277.49) as shown in Fig. 1b. The PCR amplification results suggest that the $D 12, D 61$ and $D 62$ genes had been respectively integrated into the genome of the fungus in the transformants.

Overexpressing strains were grown in complete medium for 3-4 days in $1 \mathrm{l}$ baffled flasks with $200 \mathrm{ml} \mathrm{K \& R}$ medium. Compared with the control strain, Mc-1552, Mc-D12 and Mc-D61 had a similar biomass (Fig. 2A) and lipid content (Fig. 2B), suggesting that the knockout of CarRP gene and overexpression of delta-12 or delta6-1 desaturase did not significantly affect the growth or lipid synthesis of the strains. However, Mc-D62 showed a large decrease in biomass and lipid content (Fig. 2). This indicated that the overexpression of delta-6-2 desaturase has an impact on growth and fatty acid synthesis. The possible explanation is that transformation procedure is stressful for the cells and this may cause mutations. In addition, based on the best growth and lipid production of different transformants, the strains (Mc-D12-3, Mc-D61-1, Mc-D62-2, Mc-1552-3) were selected for further analysis. The code number of the transformant is no longer marked below.

Expression levels of delta-12 and delta- 6 desaturase genes in the overexpressing strains

Real-time quantitative PCR were carried out to analyze the mRNA level of D12, D61 and D62 in the selected overexpression strains at 24,48 and $72 \mathrm{~h}$ of growth in $2 \mathrm{l}$ fermenter with K\&R medium (Fig. 3). The mRNA expression level of D12, D61 and D62 was increased, respectively, by 8 -, 7 - and 3.6 -fold at $24 \mathrm{~h}$ in overexpressing strains compared to the control, although it decreased with the incubation time. The fact that D12, D61 and D62 mRNA were maintained at elevated levels throughout the whole culture time confirmed that D12, D61 and D62 were overexpressed in the recombinant strains.

Lipid accumulation in the delta- 12 and delta- 6 desaturase overexpressing strains

Growth and lipid accumulation in D12, D61 and D62 overexpressing strains were compared to the control 


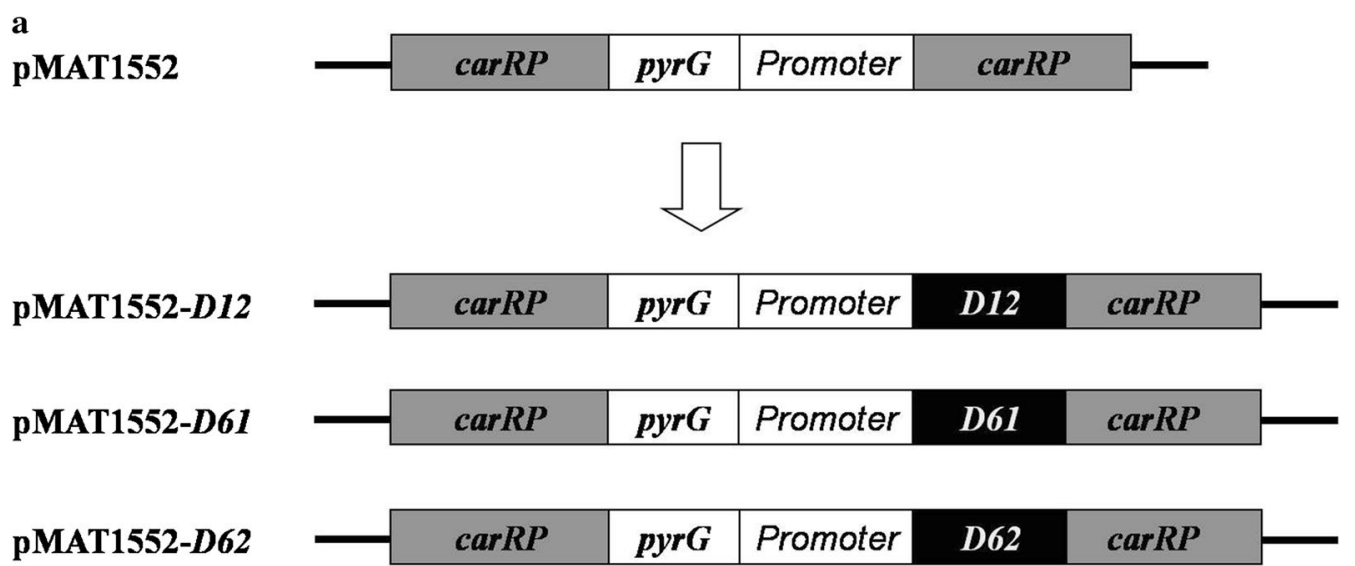

b

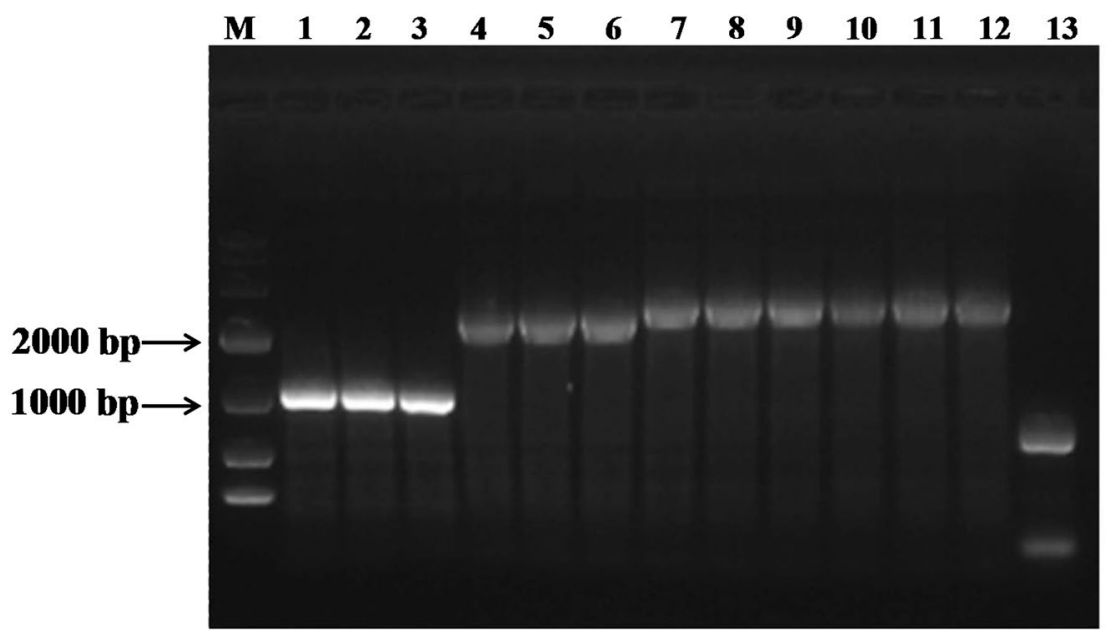

Fig. 1 Overexpression of D12, D61 and D62 genes. a Structure of plasmids pMAT1552-D12, pMAT1552-D61 and pMAT1552-D62 for D12, D61 and D62 genes overexpressing in M. circinelloides are shown. Black boxes indicate coding region of the desaturase genes. $\mathbf{b}$ PCR amplification of genome of control strain (Mc-1552), wide-type strain (M. circinelloides CBS 277.49) and desaturase gene overexpressing strains (Mc-D12, Mc-D61 and Mc-D62) with the primers P3-F and P3-R. Every three transformants in the overexpressing strains and control strain were screened and cultivated in $500 \mathrm{ml}$ flasks containing $100 \mathrm{ml} \mathrm{K \& R}$ medium for $24 \mathrm{~h}$ at $30^{\circ} \mathrm{C}$ with shaking at $150 \mathrm{rpm}$. Then, genomic DNA from these transformants was extracted and verified by PCR amplicfication. M, Gene Ruler DNA Ladder Mix, 1-3, three transformants for Mc-1552, sequentially Mc-1552-1, Mc-1552-2, Mc-15523, 4-6, three transformants for Mc-D12, sequentially Mc-D12-1, Mc-D12-2, Mc-D12-3, 7-9, three transformants for Mc-D61, sequentially Mc-D61-1, Mc-D61-2, Mc-D61-3, 10-12, three transformants for Mc-D62, sequentially Mc-D62-1, Mc-D62-2, Mc-D62-3, 13, M. circinelloides CBS 277.49

strain over $72 \mathrm{~h}$ growth in a $2 \mathrm{l}$ fermenter. Overall, the growth patterns of different strains were similar (Fig. 4a): the mycelia grew rapidly during the first $48 \mathrm{~h}$ after which the growth entered into stationary phase, and then after $72 \mathrm{~h}$, growth ceased. Except for Mc-D62, other overexpressing strains, Mc-D12 and Mc-D61, had a similar amount of biomass compared with the control strain, Mc-1552. Manipulation of the expression of delta-12 and delta-6 desaturase genes had no significantly effect on the total lipid content (Fig. 4b), whereas it had an obvious influence on the fatty acid composition (Table 1).

When compared with the control strain, Mc-1552, OA (18:1) decreased by $10 \%$ while LA (18:2) increased by $41 \%$ in Mc-D12 (Table 1), suggesting that the overexpression of delta-12 desaturase leads to the conversion of more OA to LA which should then become the substrate for GLA synthesis. However, the content of GLA in Mc-D12 decreased rather than increasing compared to the control, which suggests that delta-12 desaturase is not the rate-limiting step in GLA biosynthesis. Kelder et al. [21] has found that the $n-6$ series of fatty acids in rat $\mathrm{L}$ cells showed a downward trend upon overexpression of Mortierella alpina delta-12 desaturase which is similar to what was seen in this study. The reason for this result was likely that the increase in the membrane fluidity with the increase of LA content by delta- 12 

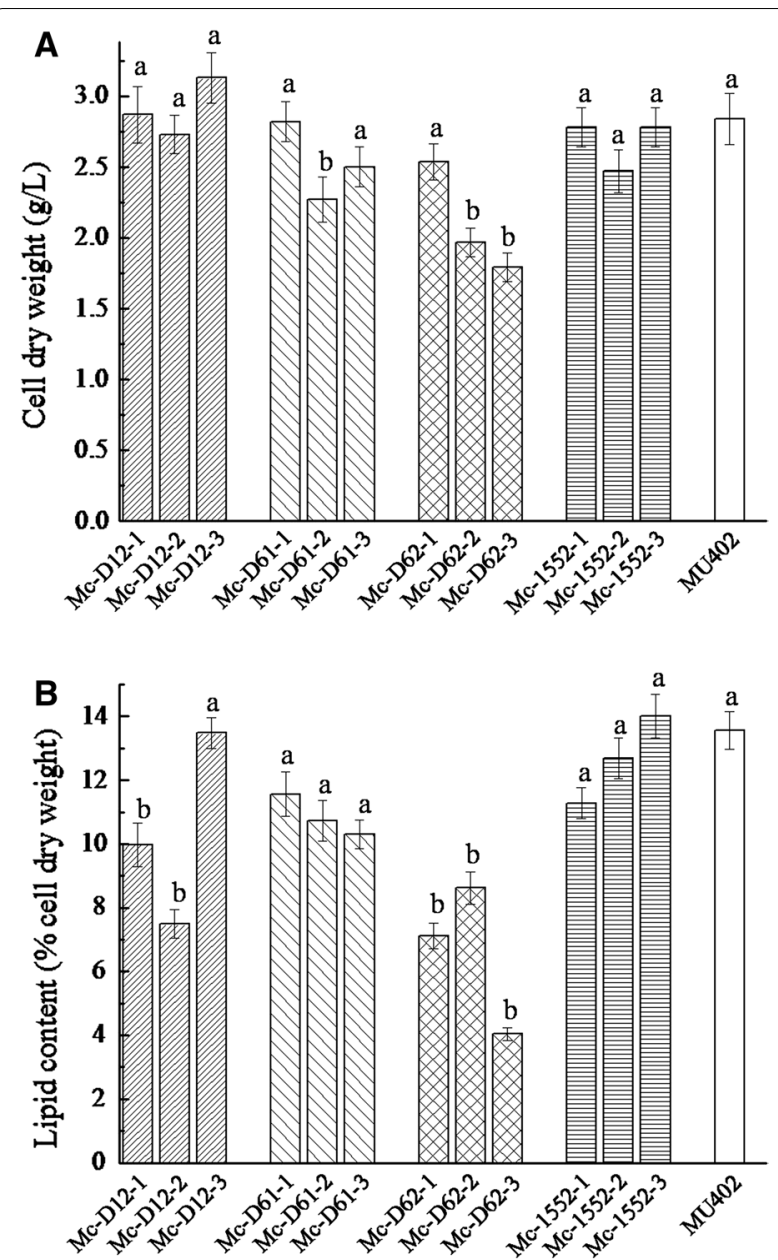

Fig. 2 Cell growth and lipid content of different overexpressing transformants. Strains were cultured in $500 \mathrm{ml}$ flasks containing $100 \mathrm{ml} \mathrm{K \& R}$ medium for $72 \mathrm{~h}$ at $30^{\circ} \mathrm{C}$ with shaking at $150 \mathrm{rpm}$. A Cell dry weight, $\mathbf{B}$ lipid content of cell dry weight. Values were mean of three independent fermentation experiments. Error bars represent the standard error of the mean

desaturase expression might cause the suppression of delta-6 desaturase.

As opposed to the overexpression of delta-12 desaturase, compared to the control strain Mc-1552, the GLA content of Mc-D61 and Mc-D62 were, respectively, increased by 38 and 19\% whilst the other fatty acids essentially remained unchanged thereby indicating that delta-6 desaturase probably plays an important role in GLA synthesis. However, the increment of GLA increase for Mc-D62 was about half of that for Mc-D61, and with a lower biomass and total lipid content. This indicated that the delta-6-2 desaturase may play a supplementing role for delta-6-1 desaturase in the synthesis of GLA. In addition, the $D 61$ overexpressing strain, Mc-D61, finally reached $180 \mathrm{mg}$ GLA/l, which was 33\% greater than that

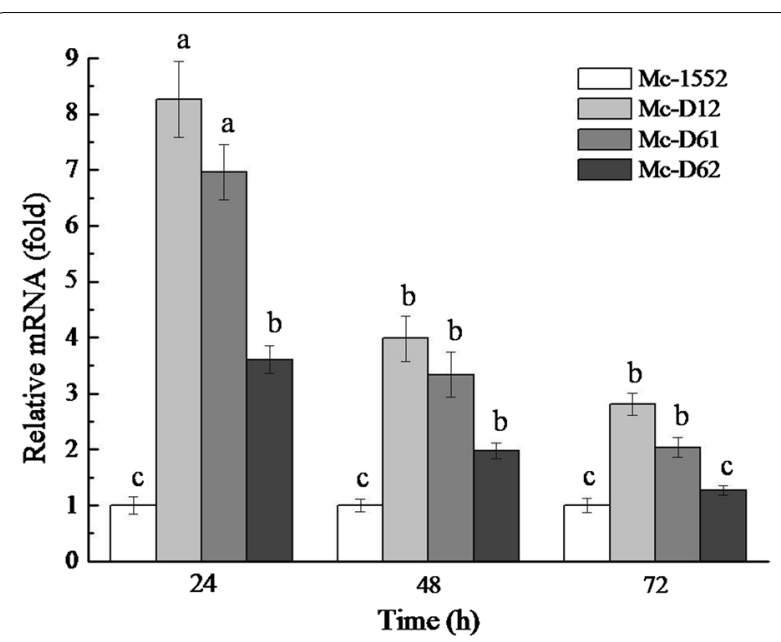

Fig. 3 Determination of expression levels of D12, D61 and D62 genes by RT-qPCR in the overexpressing strains (Mc-D12, Mc-D61 and Mc-D62), and the control strain Mc-1552. Strains were grown in a 2 I fermenter with 1.5 I K\&R medium, and the mycelium was harvested at 24,48 , and $72 \mathrm{~h}$. Total RNA of strains at different time was extracted and verified by RT-qPCR. Values were mean of three independent fermentation experiments. Error bars represent the standard error of the mean

of the control strain Mc-1552. Notably, the proportion of GLA in total lipid content for Mc-D61 reached 43\%, was much higher than that (18-19\%) in the strain of M. circinelloides used for the commercial production of GLA in the 1980s [12]. This indicates that the strain Mc-D61 may have great industrial prospects in future if the cell total lipid content can be improved.

Surprisingly in our study, the effects of these two delta- 6 fatty acid desaturases were quite different. The two delta- 6 desaturases shared only $22.8 \%$ homology and their phylogenetic positions were far apart from each other, which is in accordance with the delta- 6 genes in M. circinelloides HUT1121 [22]. Similarly, a low degree of homology particularly for fungal delta- 6 desaturases is evident when comparing the genes from Mortierella [23] and Mucor [24]. Although the sequence of delta6-2 desaturase from $M$. circinelloides was close to the delta- 6 desaturase of the high GLA-producing borage (B. officinalis), it is not efficient in GLA synthesis in $M$. circinelloides. The physiological functions of delta-6-2 desaturase need further investigation.

\section{Co-expression of delta-12 and delta- 6 desaturase genes}

To further explore the combined effect of delta- 12 and delta-6 desaturases on GLA production, co-expression of D12 and D61 were constructed in M. circinelloides. The D12 gene was inserted into the expression vector pLEU4 containing LeuA gene and then transformed into the recipient strain MU402 with the plasmid, pMAT-D61, 

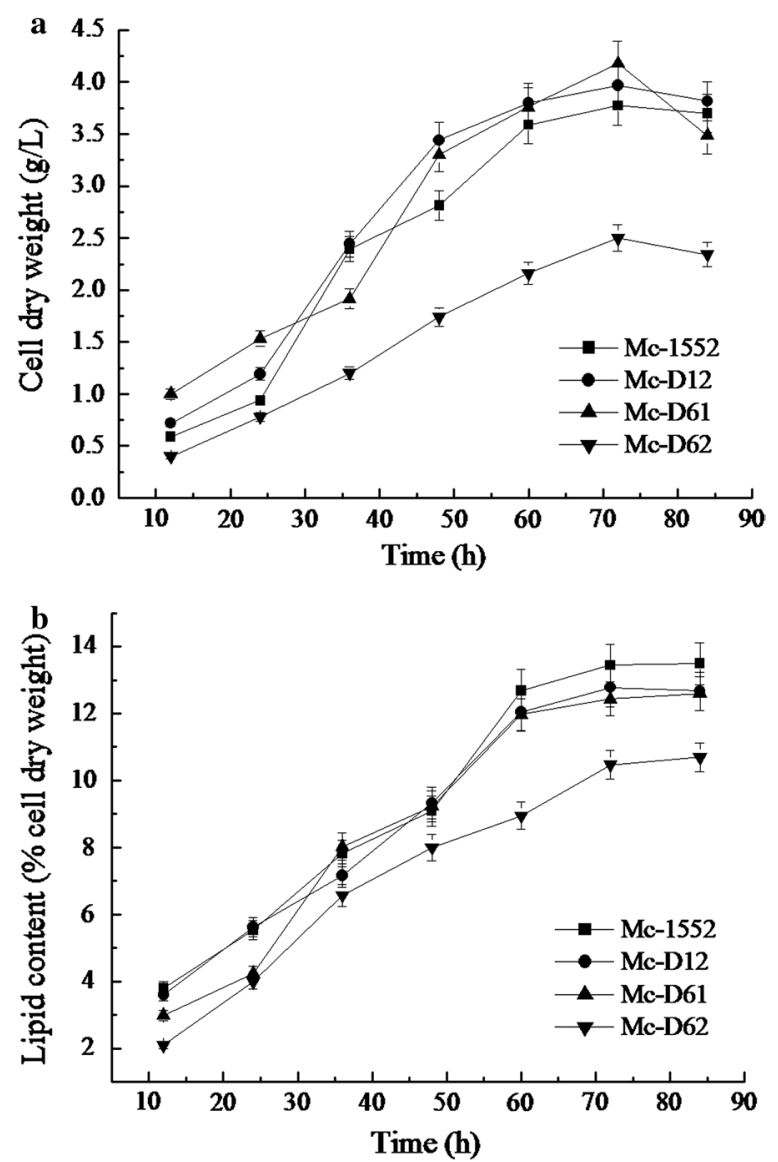

Fig. 4 Cell growth and lipid content of D12, D61 and D62 overexpressing strains. Strains were cultured in a 21 fermenter with the $1.5 \mathrm{I} \mathrm{K \& R}$ medium during $84 \mathrm{~h}$ at $30^{\circ} \mathrm{C}, \mathrm{pH} 4.5$, stirred at $500 \mathrm{rpm}$, with aeration at $0.5 \mathrm{vvm}$. In the fermentation process, biomass and lipid content were determined every $12 \mathrm{~h}$. a Cell dry weight, b lipid content of cell dry weight. Values were mean of three independent fermentation experiments. Error bars represent the standard error of the mean

overexpressing $D 61$ gene. Finally, a recombinant strain, Mc-D1261, co-expressing D12 and D61 was obtained. The mRNA expression levels of D12 and D61 were significantly increased by 8 - and 6.5 -fold in the co-expressing strain Mc-D1261 compared to the control stain Mc-1552, which was similar with the result of its single overexpression.

Growth and lipid accumulation in the co-expression strain, Mc-D1261, was examined in a 21 fermenter using K\&R medium at $72 \mathrm{~h}$ (Fig. 5A). Mc-D1261 had a slightly decrease in biomass compared with the control strain Mc-1552 but no significant change in its total lipid content. Compared with the control strain Mc-1552, the OA content of Mc-D1261 was decreased by $12 \%$, whereas the LA and GLA content of Mc-D1261 were increased by 26.5 and $15.5 \%$, respectively. Unexpectedly, the increase of GLA by co-expression of $D 12$ and $D 61$ was lower than that achieved by overexpression of the single D61. The reason for this might be that delta-12 desaturase is not rate-limiting in LA production and also, as same as the overexpression of single D12, the expression of delta-12 desaturase may have restrained the expression of delta- 6 desaturase due to the changes of membrane fluidity. Further study for the introduction of a mutation that cancels the repression of delta-12 desaturase would be needed to solve this problem.

\section{Conclusion}

Delta-12 and delta- 6 fatty acid desaturases were overexpressed by homologous recombination in the oleaginous fungus $M$. circinelloides CBS 277.49 to enhance the production of GLA. Delta- 6 desaturase overexpressing $M$. circinelloides generated in this study had up to $43 \%$ GLA in the final lipid with a final yield of $180 \mathrm{mg}$ GLA/l. These values are, respectively, 38 and 33\% higher than those in the control strain. Thus, we can conclude that delta- 6 desaturase (especially for delta-6-1 desaturase) plays an important role in GLA synthesis by M. circinelloides. The D61 overexpressing strain, Mc-D61, may have potential application in microbial GLA production.

\section{Methods}

\section{Strains, plasmids and culture conditions}

Mucor circinelloides CBS 277.49 was used as the source of delta-12 desaturase gene, D12, and two delta- 6 desaturase genes, $D 61$ and $D 62$. The leucine and uracil double auxotroph strain, MU402 [25], was used as the recipient strain in transformation experiments to overexpress the desaturase genes. Escherichia coli strain Top 10 was used for all cloning experiments and grown in lysogeny broth at $37^{\circ} \mathrm{C}$. Plasmids pMAT1552 and pLEU4 [26] were used as the cloning and expression vectors. Cultures were grown at $26{ }^{\circ} \mathrm{C}$ in YPG [27]. Media were supplemented with leucine $(20 \mu \mathrm{g} / \mathrm{ml})$ or uridine $(200 \mu \mathrm{g} / \mathrm{ml})$ when required. The $\mathrm{pH}$ was adjusted to 4.5 and 3 for mycelia and colonial growth, respectively.

The recombinant strains Mc-D12 (D12 overexpresssion strain), Mc-D61 (D61 overexpression strain), Mc-D62 (D62 overexpression strain), Mc-D1261 (D12 and D61 coexpresssion strain), and Mc-1552 (strain carrying the vector pMAT1552) as the control were initially cultivated in $500 \mathrm{ml}$ flasks containing $100 \mathrm{ml} \mathrm{K \& R}$ medium [28] for $24 \mathrm{~h}$ at $30{ }^{\circ} \mathrm{C}$ with shaking at $150 \mathrm{rpm}$ and then inoculated at $10 \%(\mathrm{v} / \mathrm{v})$ into a 21 fermenter (BioFlo/CelliGen115, New Brunswick Scientific, Edison, NJ, USA) containing $1.5 \mathrm{l} \mathrm{K \& R}$ medium modified to contain $80 \mathrm{~g}$ glucose/l. Fermenters were held at $30{ }^{\circ} \mathrm{C}$, stirred at $500 \mathrm{rpm}$, with aeration at $0.5 \mathrm{vvm}$. The $\mathrm{pH}$ was maintained at 4.5 by automatic addition of $1 \mathrm{M} \mathrm{NaOH}$ or $1 \mathrm{M}$ 
Table 1 The fatty acid composition in DELTA-12 and DELTA-6 desaturase genes overexpressing strains

\begin{tabular}{|c|c|c|c|c|c|c|}
\hline \multirow[t]{2}{*}{ Strains } & \multirow[t]{2}{*}{ Time (h) } & \multicolumn{5}{|c|}{ Fatty acid composition (relative $\%, w / w)^{a}$} \\
\hline & & $16: 0$ & 18:0 & $18: 1$ & $18: 2(\mathrm{LA})$ & $18: 3$ (GLA) \\
\hline \multicolumn{7}{|c|}{ Mc-1552 } \\
\hline & 24 & $23.1 \pm 0.5$ & $4.0 \pm 2.0$ & $23.2 \pm 8.7$ & $17.4 \pm 5.5$ & $30.0 \pm 7.1$ \\
\hline & 36 & $23.8 \pm 0.5$ & $3.6 \pm 0.1$ & $26.2 \pm 0.1$ & $14.8 \pm 1.6$ & $31.0 \pm 1.2$ \\
\hline & 48 & $21.1 \pm 0.2$ & $2.7 \pm 1.3$ & $28.3 \pm 2.6$ & $14.5 \pm 1.3$ & $31.1 \pm 2.6$ \\
\hline & 60 & $18.2 \pm 0.9$ & $2.2 \pm 0.6$ & $24.6 \pm 0.2$ & $14.3 \pm 1.8$ & $31.2 \pm 0.1$ \\
\hline & 72 & $17.1 \pm 0.6$ & $2.0 \pm 0.5$ & $24.0 \pm 3.6$ & $14.3 \pm 3.2$ & $31.2 \pm 0.4$ \\
\hline \multicolumn{7}{|l|}{ Mc-D12 } \\
\hline & 24 & $23.0 \pm 8.4$ & $3.0 \pm 0.9$ & $12.1 \pm 0.8$ & $24.0 \pm 1.4$ & $28.2 \pm 1.7$ \\
\hline & 36 & $21.5 \pm 3.1$ & $2.7 \pm 0.1$ & $20.6 \pm 3.0$ & $21.5 \pm 1.8$ & $28.1 \pm 2.3$ \\
\hline & 48 & $20.4 \pm 1.0$ & $2.2 \pm 0.3$ & $22.0 \pm 2.0$ & $20.8 \pm 1.2$ & $28.6 \pm 2.7$ \\
\hline & 60 & $19.1 \pm 0.1$ & $1.8 \pm 0.1$ & $22.4 \pm 0.3$ & $20.6 \pm 0.1$ & $28.6 \pm 0.4$ \\
\hline & 72 & $17.7 \pm 0.2$ & $1.6 \pm 0.3$ & $21.8 \pm 1.3$ & $20.1 \pm 2.1$ & $29.0 \pm 0.7$ \\
\hline \multicolumn{7}{|l|}{ Mc-D61 } \\
\hline & 24 & $22.1 \pm 0.2$ & ND & $16.5 \pm 0.1$ & $17.5 \pm 0.1$ & $41.9 \pm 0.4$ \\
\hline & 36 & $18.0 \pm 0.1$ & ND & $22.2 \pm 0.1$ & $12.3 \pm 0.0$ & $42.2 \pm 0.1$ \\
\hline & 48 & $16.8 \pm 0.2$ & $1.4 \pm 0.0$ & $22.1 \pm 0.2$ & $13.3 \pm 0.1$ & $42.6 \pm 0.0$ \\
\hline & 60 & $16.2 \pm 0.1$ & $1.3 \pm 0.3$ & $22.1 \pm 0.2$ & $14.4 \pm 0.2$ & $42.8 \pm 0.2$ \\
\hline & 72 & $14.9 \pm 0.1$ & ND & $22.1 \pm 0.2$ & $14.2 \pm 0.1$ & $43.0 \pm 0.6$ \\
\hline \multicolumn{7}{|l|}{ Mc-D62 } \\
\hline & 24 & $22.5 \pm 0.1$ & $4.2 \pm 0.0$ & $19.3 \pm 0.1$ & $16.6 \pm 0.1$ & $36.6 \pm 0.2$ \\
\hline & 36 & $22.0 \pm 0.5$ & $3.6 \pm 0.3$ & $21.5 \pm 0.2$ & $15.4 \pm 0.1$ & $36.5 \pm 0.1$ \\
\hline & 48 & $21.3 \pm 0.1$ & $3.2 \pm 0.1$ & $22.1 \pm 0.2$ & $14.4 \pm 0.1$ & $37.0 \pm 0.1$ \\
\hline & 60 & $20.2 \pm 0.2$ & $3.0 \pm 0.1$ & $22.4 \pm 0.0$ & $13.6 \pm 0.0$ & $37.0 \pm 0.1$ \\
\hline & 72 & $18.0 \pm 0.1$ & $2.7 \pm 0.3$ & $22.8 \pm 0.2$ & $13.3 \pm 0.1$ & $37.1 \pm 0.6$ \\
\hline
\end{tabular}

ND not detected

a Strains were cultured in a 2 I fermenter with the 1.5 I Kendrick medium during $72 \mathrm{~h}$. The fatty acid composition were displayed at different point times. The values are means \pm standard deviations of two independent experiments

$\mathrm{HCl}$, and medium was supplied with leucine $(20 \mu \mathrm{g} / \mathrm{ml})$ or uracil $(200 \mu \mathrm{g} / \mathrm{ml})$ when required.

\section{Plasmids construction}

The vector, pMAT1552, which was used for construction of D12, D61 and D62 overexpression plasmids, contains only a XhoI restriction site between its promoter and homology arm CarRP sequence. To facilitate subsequent cloning screening, a CarRP sequence was amplified by using primers Car-F/R (Additional file 1: Table S1), with another $X b a \mathrm{I}$ restriction site inserted between the XhoI and SacII sites of original CarRP fragment. The amplification product was isolated and ligated into the vector pMD19T-simple to generate an intermediate vector pMD19T-CarRP.

The genes, $D 12, D 61$ and $D 62$, were isolated by PCR amplification from the genome of $M$. circinelloides CBS 277.49 with separate primers D12-F/R, D61-F/R and D62-F/R (Additional file 1: Table S1) that introduced the $X h o \mathrm{I}$ and $X b a \mathrm{I}$ restriction sites, respectively, at the $5^{\prime}$ - and $3^{\prime}$-ends of desaturase genes. The products were digested by $X h o I$ and $X b a I$ and ligated into the similarly digested intermediate vector $\mathrm{pMD} 19 \mathrm{~T}-\operatorname{CarRP}$. The ligation mixture was used to transform chemically competent $E$. coli Top 10 cells. The plasmids isolated from these transformants were verified by restriction analysis and the gene sequences were confirmed by DNA sequencing. The plasmids with the correct sequences of D12, D61 and D62 were named pMD19T-CarRP-D12, pMD19TCarRP-D61 and pMD19T-CarRP-D62, respectively. Because D12 and D61 genes contain one internal XhoI site, site-directed mutagenesis was performed to eliminate these sites while keeping the encoded amino acid unchanged. The resulting plasmids were digested with XhoI and EcoRI and ligated into the similarly digested expression vector pMAT1552. The ligation mixture was used to transform chemically competent $E$. coli Top 10 cells. The plasmids isolated from these transformants were verified by restriction analysis and the gene sequences were confirmed again by DNA sequencing. 

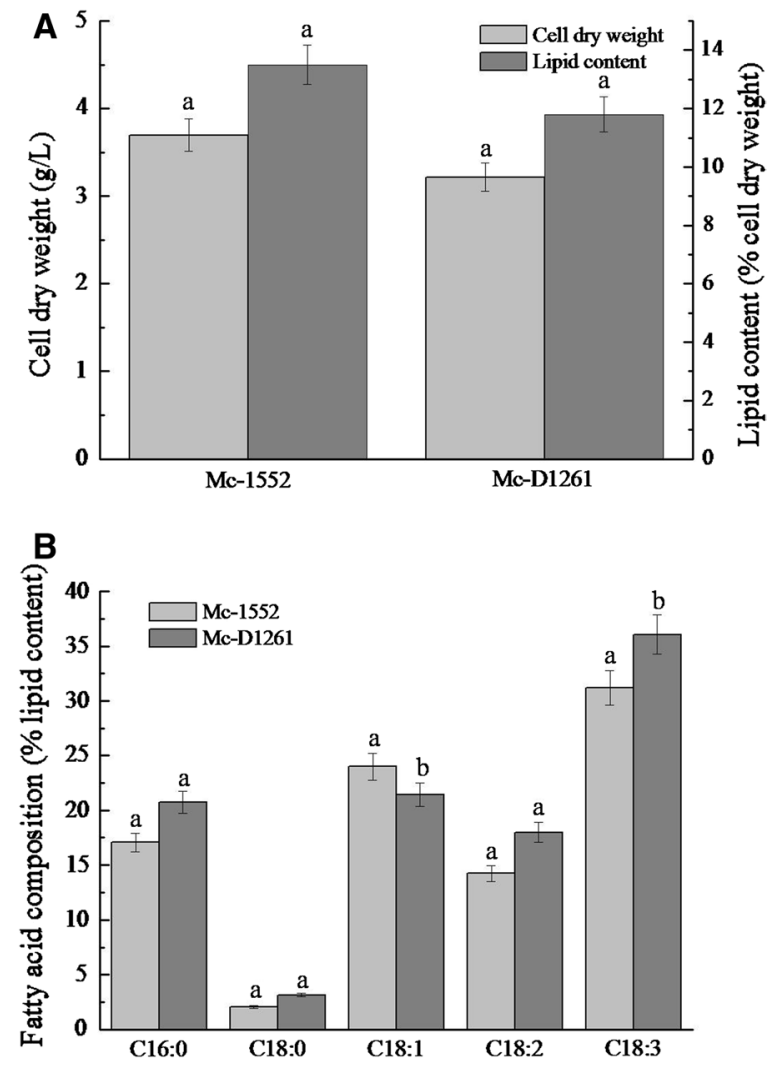

Fig. 5 Cell growth and lipid accumulation in D12 and D61 Coexpressing strain (Mc-D1261) and the control strain Mc-1552. Strains were cultured in a $2 \mathrm{I}$ fermenter with the $1.5 \mathrm{I}$ K\&R medium for $72 \mathrm{~h}$ at $30^{\circ} \mathrm{C}, \mathrm{pH} 4.5$, stirred at $500 \mathrm{rpm}$, with aeration at $0.5 \mathrm{vvm}$. A Cell dry weight and lipid content of cell dry weight, $\mathbf{B}$ the fatty acid composition. Values were mean of three independent fermentation experiments. Error bars represent the standard error of the mean

The plasmids with the correct sequences of D12, D61 and D62 were named pMAT-D12, pMAT-D61 and pMAT-D62, respectively. Transformation was carried out by electroporation-mediated procedure as described previously [29].

To achieve the co-expression of $D 12$ and $D 61$, another D12 gene expression plasmid was constructed using the vector pLEU4. The D12 gene was isolated by PCR amplification from the plasmid pMAT-D12 and then cloned into the expression vector pLEU4 with primers D12'-F/R (Additional file 1: Table S1). The amplification product was digested by KasI and SalI and ligated into the similarly digested expression vector pLEU4. The ligation mixture was used to transform chemically competent $E$. coli Top 10 cells. The plasmids isolated from these transformants were verified by restriction analysis and the gene sequences were confirmed by DNA sequencing. The plasmids with the correct sequences of D12 was named
pLEU-D12. Then, the plasmids pLEU-D12 and pMATD61 were simultaneously transformed into competent MU402 cells.

\section{Gene expression and RT-qPCR analysis}

D12, D61 and D62 overexpressing transformants were cultivated in YNB for 3 days before extracting the genomic DNA. The mycelia was harvested by suction filtration and washed twice with sterile water. The genomic DNA was extracted by following the protocols of the DNA Quick Plant System (Tiangen). PCR amplication was used to verify whether $D 12, D 61$ or $D 62$ had integrated into the genome of $M$. circinelloides with the universal primers P3-F/R (Additional file 1: Table S1).

For reverse transcription-quantitative PCR (RT-qPCR) analysis, strains were grown in a 21 fermenter with $K \& R$ medium, and the mycelium was harvested at 24,48 , and $72 \mathrm{~h}$. Total RNA of $M$. circinelloides was extracted with Trizol after grinding under liquid $\mathrm{N}_{2}$ and reverse-transcribed using the Prime ScriptRT reagent kit (Takara) according to the manufacturer's instructions. RT-qPCR was performed using primers (Additional file 1: Table S1) on CFX Connect Real-Time System (Bio-Rad) with iTaq Universal SYBR Green PCR Supermix (Bio-Rad) according to the manufacturer's instruction. The mRNA expression level was normalized to levels of $18 \mathrm{~S}$ rRNA mRNA, and the results were expressed as relative expression levels. The data were quantified by the method of $2^{-\Delta \Delta C t}$.

\section{Determination of cell dry weight and fatty acid analysis}

Biomass was harvested on a weighed filter paper by filtration through a Buchner funnel under reduced pressure and washed three times with distilled water, frozen overnight at $-80{ }^{\circ} \mathrm{C}$ and then freeze-dried. The weight of the biomass was determined gravimetrically.

Biomass was collected by filtration and dried by lyophilizer. $20 \mathrm{mg}$ dry weight was taken for cell fatty acids extraction. Pentadecanoic acid (15:0) was added into the freeze-dried cells as an internal standard. The total fatty acids were extracted with chloroform/methanol $(2: 1, \mathrm{v} / \mathrm{v})$ and methylated with $10 \%(\mathrm{v} / \mathrm{v})$ methanolic $\mathrm{HCl}$ at $60{ }^{\circ} \mathrm{C}$ for $3 \mathrm{~h}$. The resultant fatty acid methyl esters were extracted with n-hexane and were analyzed by GC equipped a $30 \mathrm{~m} \times 0.32 \mathrm{~mm}$ DB-Waxetr column with $0.25 \mu \mathrm{m}$ film thickness. The program was as follows: $120^{\circ} \mathrm{C}$ for $3 \mathrm{~min}$, ramp to $200^{\circ} \mathrm{C}$ at $5^{\circ} \mathrm{C}$ per min, ramp to $220^{\circ} \mathrm{C}$ at $4{ }^{\circ} \mathrm{C}$ per min, hold $2 \mathrm{~min}$.

\section{Statistical analysis}

A statistical analysis was carried out using SPSS 16.0 for Windows (SPSS Inc. Chicago, IL). The mean values and the standard error of the mean were calculated from the data obtained from three independent experiments. The 
differences between the means of the test were evaluated by Student's $t$ test, and $P<0.05$ was considered as significantly different.

\section{Additional file}

Additional file 1: Table S1. Primer sequences used in this study.

\section{Abbreviations}

GLA: $\gamma$-linolenic acid; PUFA: polyunsaturated fatty acid; GM: genetically modified; OA: oleic acid; LA: linoleic acid; GC: gas chromatography.

\section{Authors' contributions}

$Y Z$ and $X L$ carried out the experiments and drafted the manuscript. $H Z, V G, Y S$ and CR participated in the experimental design and reviewed the manuscript. YS and CR conceived the study and reviewed the final manuscript. All authors read and approved the final manuscript.

\section{Author details}

${ }^{1}$ Colin Ratledge Center for Microbial Lipids, School of Agricultural Engineering and Food Science, Key Laboratory of Shandong Provincial Universities for Technologies in Functional Agricultural Products, Shandong University of Technology, 266 Xincun West Road, Zibo, Shandong 255000, People's Republic of China. ${ }^{2}$ School of Food Science and Technolgy, Jiangnan University, 1800 Lihu Avenue, Wuxi, Jiangsu 214122, People's Republic of China. ${ }^{3}$ Departamento de Genéticay Microbiologia (Unidad ASOCIADA al IQFR-CSIC), Facultad de Biologi, Universidad de Murcia, 30071 Murcia, Spain. ${ }^{4}$ Department of Biological Sciences, University of Hull, Hull, UK.

\section{Acknowledgements}

This work was supported by the National Natural Science Foundation of China (Nos. 31670064 and 31271812), Taishan Industry Leading Talent Project, Science and Technology Project of Shandong College (No. J16LE20), China Postdoctoral Science Foundation (Nos. 2017M612305 and 2015T80735), Doctor and Postdoctoral Foundation of Shandong University of Technology (Nos. 4041/415047 and 4041/516002).

\section{Competing interests}

The authors declare that they have no competing interests.

\section{Availability of data and materials}

All data generated or analysed during this study are included in this published article [and its Additional file].

\section{Publisher's Note}

Springer Nature remains neutral with regard to jurisdictional claims in published maps and institutional affiliations.

Received: 9 March 2017 Accepted: 7 June 2017

Published online: 21 June 2017

\section{References}

1. Lu H, Zhu Y. Screening and molecular identification of overproducing gamma-linolenic acid fungi and cloning the delta 6-desaturase gene. Biotechnol Appl Biochem. 2015;62(3):316-22.

2. Horrobin DF. Nutritional and medical importance of gamma-linolenic acid. Prog Lipid Res. 1992;31(2):163-94.

3. Fan YY, Chapkin RS. Importance of dietary gamma-linolenic acid in human health and nutrition. J Nutr. 1998:128(9):1411-4

4. Kim DH, Yoo TH, Lee SH, Kang HY, Nam BY, Kwak SJ, Kim JK, Park JT, Han SH, Kang SW. Gamma linolenic acid exerts anti-inflammatory and anti-fibrotic effects in diabetic nephropathy. Yonsei Med J. 2012;53(6):1165-75.
5. Wan X, Zhang Y, Wang P, Huang F, Chen H, Jiang M. Production of gamma-linolenic acid in Pichia pastoris by expression of a delta- 6 desaturase gene from Cunninghamella echinulata. J Microbiol Biotechnol. 2009;19(10):1098-102.

6. Wan X, Zhang Y, Wang P, Jiang M. Molecular cloning and expression analysis of a delta 6-fatty acid desaturase gene from Rhizopus stolonifer strain YF6 which can accumulate high levels of gamma-linolenic acid. J Microbiol. 2011:49(1):151-4.

7. Dyer JM, Stymne S, Green AG, Carlsson AS. High-value oils from plants. Plant J. 2008;54(4):640-55.

8. Nykiforuk CL, Shewmaker C, Harry I, Yurchenko OP, Zhang M, Reed C, Oinam GS, Zaplachinski S, Fidantsef A, Boothe JG, Moloney MM. High level accumulation of gamma linolenic acid (C18:3 Delta 6.9,12 cis) in transgenic safflower (Carthamus tinctorius) seeds. Transgenic Res. 2012;21(2):367-81.

9. Knauf VC, Shewmaker C, Flider F, Emlay D, Rey E. Safflower with elevated gamma-linolenic acid. US Patent 7893321 B2. 2011.

10. Fakas S, Certik M, Papanikolaou S, Aggelis G, Komaitis M, Galiotou-Panayotou M. Gamma-linolenic acid production by Cunninghamella echinulata growing on complex organic nitrogen sources. Bioresour Technol. 2008;99(13):5986-90.

11. Ratledge C. Microorganisms as sources of polyunsaturated fatty acids. In: Gunstone FD, editor. Structured and Modified Lipids. New York, USA: Marcel Dekker, Inc. 2001. p. 351-99.

12. Ratledge C. Microbial production of gamma-linolenic acid. In: Akoh CC, editor. Handbook of functional lipids. Boca Raton: CRC Press; 2006. p. 19-45.

13. Cui J, He S, Ji X, Lin L, Wei Y, Zhang Q. Identification and characterization of a novel bifunctional delta(12)/delta(15)-fatty acid desaturase gene from Rhodosporidium kratochvilovae. Biotechnol Lett. 2016;38(7):1155-64.

14. Passorn S, Laoteng K, Rachadawong S, Tanticharoen M, Cheevadhanarak S. Heterologous expression of Mucor rouxii delta(12)-desaturase gene in Saccharomyces cerevisiae. Biochem Biophys Res Commun. 1999;263(1):47-51.

15. Qiao K, Imam Abidi SH, Liu H, Zhang H, Chakraborty S, Watson N, Kumaran Ajikumar P, Stephanopoulos G. Engineering lipid overproduction in the oleaginous yeast Yarrowia lipolytica. Metab Eng. 2016;29:56-65.

16. Chen G, Qu S, Wang Q, Bian F, Peng Z, Zhang Y, Ge H, Yu J, Xuan N, Bi Y, He Q. Transgenic expression of delta- 6 and delta- 15 fatty acid desaturases enhances omega-3 polyunsaturated fatty acid accumulation in Synechocystis sp. PCC6803. Biotechnol Biofuels. 2014;7(1):32.

17. Ratledge C, Wynn JP. The biochemistry and molecular biology of lipid accumulation in oleaginous microorganisms. Adv Appl Microbiol. 2002;51:1-51.

18. Tang $X$, Chen H, Chen YQ, Chen W, Garre V, Song Y, Ratledge C. Comparison of biochemical activities between high and low lipid-producing strains of Mucor circinelloides: an explanation for the high oleaginicity of strain WJ11. PLoS ONE. 2015;10(6):e0128396.

19. Tang $X$, Zhang $H$, Chen $H$, Chen YQ, Chen W, Song Y. Effects of 20 standard amino acids on the growth, total fatty acids production, and gamma-linolenic acid yield in Mucor circinelloides. Curr Microbiol. 2014;69(6):899-908.

20. Rodriguez-Frometa RA, Gutierrez A, Torres-Martinez S, Garre V. Malic enzyme activity is not the only bottleneck for lipid accumulation in the oleaginous fungus Mucor circinelloides. Appl Microbiol Biotechnol. 2013;97(7):3063-72

21. Kelder B, Mukeji P, Kirchner S, Hovanec G, Leonard AE, Chuang LT, Kopchick JJ, Huang YS. Expression of fungal desaturase genes in cultured mammalian cells. Mol Cell Biochem. 2001;219(1-2):7-11.

22. Michinaka Y, Aki T, Shimauchi T, Nakajima T, Kawamoto S, Shigeta S, Suzuki O, Ono K. Differential response to low temperature of two Delta6 fatty acid desaturases from Mucor circinelloides. Appl Microbiol Biotechnol. 2003;62(4):362-8.

23. Sakuradani E, Kobayashi M, Shimizu S. Delta6-fatty acid desaturase from an arachidonic acid-producing Mortierella fungus. Gene cloning and its heterologous expression in a fungus, Aspergillus. Gene. 1999;238(2):445-53.

24. Laoteng K, Mannontarat R, Tanticharoen M, Cheevadhanarak S. Delta(6)-desaturase of Mucor rouxii with high similarity to plant delta(6)desaturase and its heterologous expression in Saccharomyces cerevisiae. Biochem Biophys Res Commun. 2000;279(1):17-22. 
25. Nicolas FE, de Haro JP, Torres-Martinez S, Ruiz-Vazquez RM. Mutants defective in a Mucor circinelloides dicer-like gene are not compromised in siRNA silencing but display developmental defects. Fungal Genet Biol. 2007:44(6):504-16.

26. Roncero MI, Jepsen LP, Stroman P, van Heeswijck R. Characterization of a leuA gene and an ARS element from Mucor circinelloides. Gene. 1989;84(2):335-43.

27. Bartnicki-Garcia S, Nickerson WJ. Nutrition, growth, and morphogenesis of Mucor rouxii. J Bacteriol. 1962;84:841-58.
28. Kendrick A, Ratledge C. Desaturation of polyunsaturated fatty acids in Mucor circinelloides and the involvement of a novel membrane-bound malic enzyme. Eur J Biochem. 1992;209(2):667-73.

29. Torres-Martinez S, Ruiz-Vazquez RM, Garre V, Lopez-Garcia S, Navarro E, Vila A. Molecular tools for carotenogenesis analysis in the zygomycete Mucor circinelloides. Methods Mol Biol. 2012;898:85-107.

\section{Submit your next manuscript to BioMed Central and we will help you at every step:}

- We accept pre-submission inquiries

- Our selector tool helps you to find the most relevant journal

- We provide round the clock customer support

- Convenient online submission

- Thorough peer review

- Inclusion in PubMed and all major indexing services

- Maximum visibility for your research

Submit your manuscript at

www.biomedcentral com/submit 